\title{
Design und Modellierung eines 3D-Nanotasters
}

\author{
Jan Thiesler ${ }^{1}$, Gaoliang Dai', Rainer Tutsch ${ }^{2}$ \\ ${ }^{1}$ Physikalisch-Technische Bundesanstalt, Bundesallee 100, Deutschland \\ ${ }^{2}$ Technische Universität Braunschweig, IPROM, Schleinitzstraße 20, Deutschland
}

\section{Zusammenfassung}

In der Nanometrologie werden Strukturen immer kompakter und komplexer, dabei basiert deren Funktionalität auf genau bekannten dreidimensionalen geometrischen Abmessungen und definierter Rauigkeit der Oberfläche. So ist eine Miniaturisierung in der Halbleiterindustrie vielfach durch Wandrauigkeiten (LER = line-edge roughness) limitiert und eine genaue Kenntnis des Fertigungsprozesses ist notwendig. Das vorgestellte Design eines 3D-Nanotasters basiert auf dem Prinzip eines Biegebalkens eines CD-AFM Cantilevers. Dieser strukturierte Biegebalken beinhaltet zwei Festkörpergelenke, ist hinsichtlich isotroper Steifigkeit $(<1 \mathrm{~N} / \mathrm{m})$ und 3D-Sensitivität optimiert, besitzt eine erste Eigenfrequenz von $59,3 \mathrm{kHz}$ und ist als Taster für Spitzendurchmesser unter $100 \mathrm{~nm}$ konzipiert.

Keywords: Nanotaster, CD-Cantilever, 3D-AFM, Critical Dimension, $\mu$ KMG

\section{Einleitung}

Seit der Erfindung des Rasterkraftmikroskopes durch G. Binnig et al. 1985/86 [1] hat sich das Design der mit der Probe wechselwirkenden Sonde als Biegebalken, zur Detektion von Oberflächentopographien im Nanometerbereich für Rastersondenmikroskope, durchgesetzt. Dieser Biegebalken oder Cantilever verformt sich elastisch durch die Kräfte der wechselwirkenden Oberflächenpotentiale von der Spitze der Sonde und Probe. Diese Verformung kann interferometrisch, kapazitiv, piezoresistiv oder über ein Lichtzeigerprinzip ausgelesen werden. Dabei ist der klassische Cantilever (Abb. 1) ein 1D-Sensor, der lediglich die Kräfte senkrecht zu seiner Längsachse bei glatter Probenoberfläche als eine Biegung zeigt.

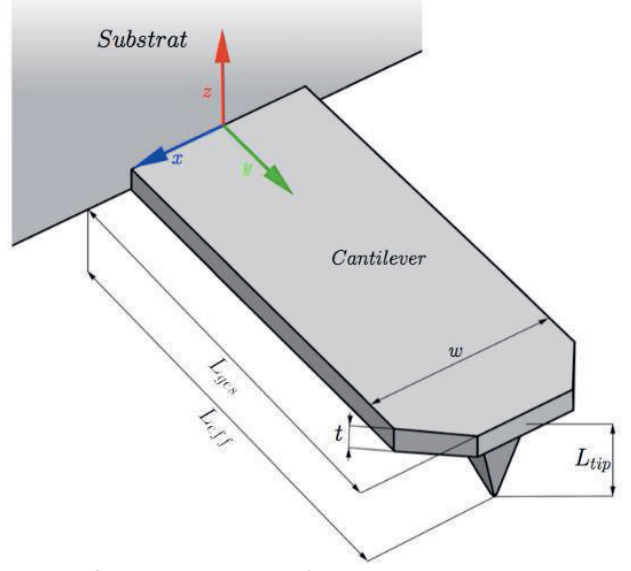

Abb. 1: Skizze eines Cantilevers mit Variablen (Breite $=w$, Dicke $=t$, effektive Länge $=L_{\text {eff }}$ und Spitzenlänge $=L_{\text {tip }}$ ) und Koordinatensystem
Spezielle Cantilever mit am Ende verbreiteter Spitze für CD-AFMs, im Folgenden als CDCantilever $(C D=$ critical dimension) (Abb. 2) bezeichnet, sind mit dem entsprechenden Tastkopf des Rasterkraftmikroskops, in der Lage als 2D-Sensor zu arbeiten. Es wird die Biegung und Torsion des Cantilevers detektiert $[2,3]$.

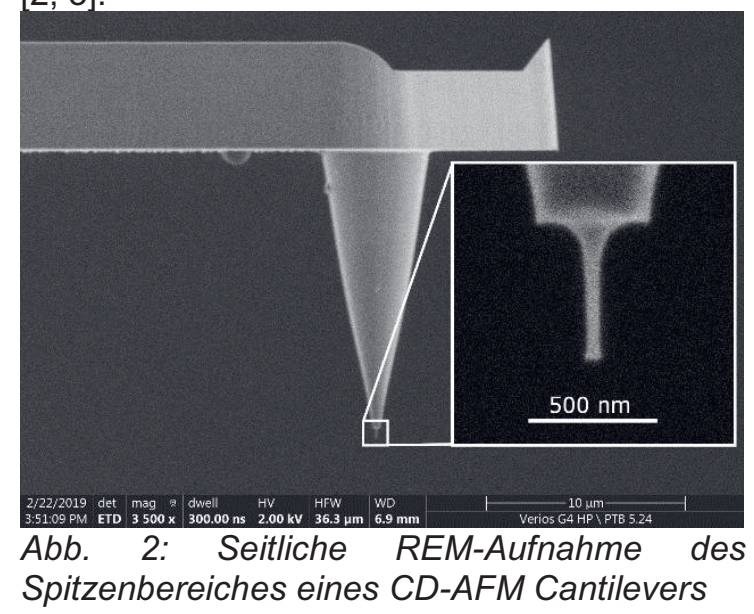

Die CD-Cantilever weisen weiterhin eine am Ende ausgestellte Spitze mit Durchmessern von $850 \mathrm{~nm}$ bis $15 \mathrm{~nm}$ auf $[4,5]$, die es ermöglicht Hinterschneidungen, an den sonst nicht zugänglichen Seitenwänden der Probenoberfläche, abzubilden. Jedoch besitzen diese CD-Cantilever eine um Größenordnungen geringere Sensitivität, durch Kombination der inversen Steifigkeiten von Spitze und Cantilever, bei seitlichem Antasten der Spitze und sind anisotrop. Diese von CDAFM oder auch 3D-AFM benutzten Cantilever, sind in der Halbleiterindustrie weit verbreitet 
und werden zum Messen von Masken für die Lithographie, Rauheitsmessung und Prozesskontrolle, sowie für die geometrische Überprüfung komplexer Nanostrukturen verwendet. Dabei ist zu beachten, dass das bezeichnete 3D-AFM keine echt 3Dmessenden Eigenschaften besitzt [2, 6]. Der verwendete Cantilever kann lediglich zwei orthogonale Richtungen auflösen, durch Konturschnitte wird ein dreidimensionaler Eindruck der Oberfläche erzeugt [6]. Da die fertigungsbedingt möglichen Geometrien von Mikro- und Nanostrukturen immer komplexer werden ist ein Messgerät gefordert, dass ähnlich einer Mikro-KoordinatenMessmaschine ( $\mathrm{K} \mathrm{KMG})$ dreidimensionale Strukturen auch dreidimensional antasten kann. Jedoch besitzen $\mu \mathrm{KMGs}$ derzeit Sonden mit einigen Mikrometern Spitzendurchmesser [715], der ein Antasten kleiner Radien und Strukturbreiten im unteren Mikrometerbereich verhindert und ein Messen von beispielsweise Mikroverzahnungen unmöglich macht. Um diesen Umständen zu begegnen wird derzeit an echt 3D-messenden NanoPositioniermaschinen gearbeitet beispielsweise die NPMM-200 [16, 17]. Bedauerlicherweise sind Geräte bezüglich der taktilen Messung mit Spitzendurchmessern im Nanometerbereich noch in der Entwicklung.

Es gilt somit einen 3D-Nanotaster zu entwickeln, der Eigenschaften wie quasiisotrope Steifigkeit des $\mu \mathrm{KMG}$-Taster beinhaltet und gleichzeitig die Vorteile eines 3D-AFMs mit den geringen Spitzendurchmessern und hoher Eigenfrequenz der CD-Cantilever vereint.

Im Interesse der Zugänglichkeit für komplex geformte Proben, wird auf ein CantileverDesign für die Sonde, der 3D-Nanotaster, nicht verzichtet. Weiterhin gilt es, ein ausgewogenes Verhältnis für die Sensitivität und Selektivität der Detektionsrichtungen zu erhalten und auf ein prototypengerechtes Design zu achten, das mit einer lonenfeinstrahlanlage hergestellt werden kann.

\section{Design des Nanotasters}

Ausgehend von einem klassischen rechteckförmigem Cantilever mit rechteckigem Querschnitt, zeigt dieser eine Anisotropie bezüglich seiner Komponenten $\mathbf{c}_{\mathrm{ii}}$ mit $(\mathrm{i}=\mathrm{x}, \mathrm{y}, \mathrm{z})$ der inversen Steifigkeitsmatrix für kleine Auslenkungen, $\underline{\underline{\boldsymbol{C}}}_{c}$ siehe GI. (1). Das Verhältnis dieser Anisotropie liegt für $(x / y / z)$ in der Größenordnung von (20/20/1). Weiterhin besitzt die symmetrische Matrix $\underline{\boldsymbol{C}}_{c}$ Elemente auf den Nebendiagonalen die ungleich Null sind. Diese Elemente haben ein Übersprechen der bisher nicht detektierbaren y-Richtung auf die zRichtung (und umgekehrt) zur Folge. Dies führt zur Fehlinterpretation bei dem Bestimmen geometrischer Abmessungen und ist für den 3D-Nanotaster zu minimieren.

Die Ableitung der inversen Steifigkeitsmatrix ist die Matrix für die Auslenkwinkel des Cantilevers $\underline{\boldsymbol{A}_{c}}$ siehe GI. (2). Diese zeigt in Abhängigkeit der wirkenden Kräfte oder Verschiebung der Spitze eine Winkeländerung des vorderen freien Endes des Cantilevers. Die Winkeländerung wird über einen Lichtzeiger detektiert.

$$
\underline{\boldsymbol{C}_{c}}=\left[\begin{array}{ccc}
\frac{2 L_{t i p}^{2}}{L_{e f f}^{2}}+\frac{t^{2}}{w^{2}} & 0 & 0 \\
0 & \frac{3 L_{\text {tip }}^{2}}{L_{\text {eff }}^{2}} & \frac{3 L_{t i p}}{2 L_{\text {eff }}} \\
0 & \frac{3 L_{\text {tip }}}{2 L_{\text {eff }}} & 1
\end{array}\right] c ; c=\frac{4 L_{\text {eff }}^{3}}{E w t^{3}}
$$

$$
\underline{\boldsymbol{A}} \boldsymbol{c}=\left(\begin{array}{l}
\alpha \\
\beta \\
\gamma
\end{array}\right)=\left[\begin{array}{ccc}
0 & \frac{3 L_{t i p}}{L_{e f f}^{2}} & \frac{3}{2 L_{e f f}} \\
\frac{2 L_{t i p}}{L_{e f f}^{2}} & 0 & 0 \\
\frac{3 t^{2}}{2 w^{2} L_{e f f}} & 0 & 0
\end{array}\right]\left(\begin{array}{l}
F_{x} \\
F_{y} \\
F_{z}
\end{array}\right) c
$$

Durch Kombination mit der inversen Steifigkeit einer biegeweichen Spitze $\underline{\underline{\boldsymbol{C}}}$ s (siehe GI. (2)) kann die Isotropie hergestellt werden, jedoch skaliert die Größenordnung der Spitze mit ihrer Steifigkeit. Mit abnehmendem Durchmesser, bei gleichem Aspektverhältnis (üblicherweise $6: 1)$, nimmt ebenfalls die Steifigkeit ab. Dies hat zur Folge das mit kleineren Spitzendurchmessern die Sensitivität abnimmt, beziehungsweise die minimal detektierbare Verschiebung zunimmt, welches die Unsicherheit vergrößert, da die Verschiebung der Spitze nicht detektiert wird, siehe GI. (4).

$\underline{\underline{\boldsymbol{C}}} \boldsymbol{s}=\left[\begin{array}{ccc}\frac{64 L_{s}^{3}}{3 E d^{4} \pi} & 0 & 0 \\ 0 & \frac{64 L_{s}^{3}}{3 E d^{4} \pi} & 0 \\ 0 & 0 & \frac{4 L_{s}}{E d^{2} \pi}\end{array}\right]$

$\underline{A}_{s}=0$

Somit wird ein Design (Abb. 3) vorgestellt, bei dem die Nebendiagonalen nach einer Optimierung Null sind und die kombinierte inverse Steifigkeit für die Diagonalelemente $c_{x x} \approx$ $c_{y y} \approx c_{z z}$ ist, ein Cantilever mit isotroper Steifigkeit. Dadurch wird ein unkontrolliertes Rutschen der Tastspitze des 3D-Nanotasters für ein Antasten einer Oberfläche in Normalenrichtung vermieden. Es wird ein an der yz-Ebene symmetrisches Design gewählt, da hier unter Berücksichtigung der zy- und yzKomponenten die Nebendiagonalen bereits in guter Näherung Null sind. Das vorgestellte Design weist zwei Festkörpergelenke auf, die die Steifigkeit der Struktur beeinflussen. Der zweite Hals besitzt ein negatives Vorzeichen 
bezüglich zy- und yz-Komponenten und kann somit zu deren Minimierung beitragen. Weiterhin lässt sich der nicht detektierbare, aber für ein von Übersprechen von $\alpha$ und $\beta$ verantwortlicher Winkel y reduzieren (GI. 2). Die 3-Selektivität wird durch ein mit dem Reduzieren der Nebendiagonalen einhergehenden Minimieren der Auslenkung $\alpha\left(F_{z}\right)$ erreicht.

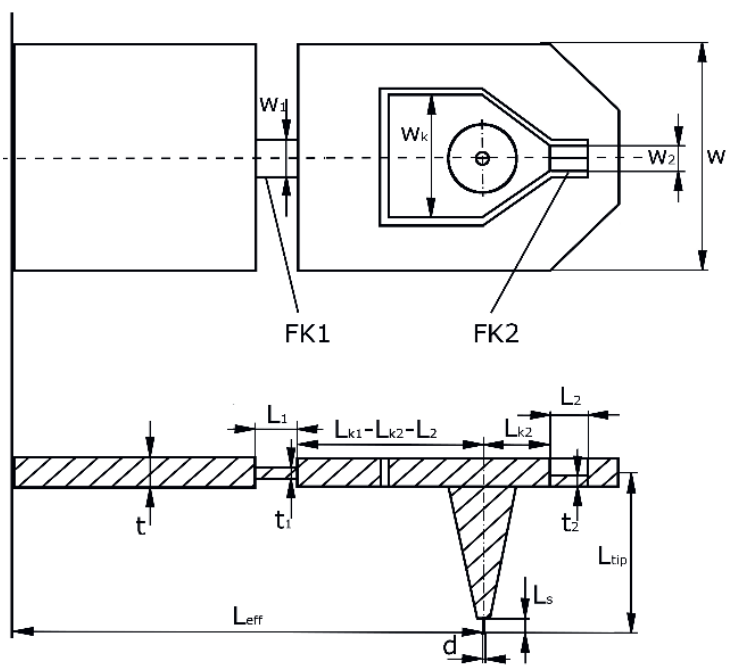

Abb. 3: Skizze des Designs des 3DNanotasters mit Variablen für die Berechnung (FK = Festkörpergelenk).

Der Kopfbereich des 3D-Nanotasters zeigt für eine Verschiebung in z-Richtung ein Heben und Senken (Auslesemöglichkeit: Interferometer) und für Verschiebungen bzw. Kräfte in xRichtung oder $y$-Richtung eine Winkeländerung (Auslesemöglichkeit: Quadranten-Photodiode). Durch die Miniaturisierung sind Eigenfrequenzen von einigen $10 \mathrm{kHz}$ bei Steifigkeiten von $1 \mathrm{~N} / \mathrm{m}$ zu erwarten.

\section{Modellbildung}

Das oben in Abb. 3 vorgestellte Design zeigt den 3D-Nanotaster mit zwei Festkörpergelenken. Dabei besitzt das Gelenk 1 einen rechteckigen Querschnitt und Gelenk 2 aus fertigungstechnischen Gründen der lonenfeinstrahlanlage einen dreieckigen Querschnitt. Die Modellierung erfolgt mit Hilfe der Euler-Bernoulli Balkentheorie, da in den interessanten Bereichen die Querkräfte im Vergleich zu den anliegenden Momenten klein sind und elastische Auslenkungen im Bereich einiger zehn $\mu$ rad zu erwarten sind. Es wird das Superpositionsprinzip für die Matrizen der inversen Steifigkeiten und der Auslenkwinkel angewendet (im Folgenden nicht weiter betrachtet). Dabei wird von einer festen Einspannung $\underline{\underline{C}}_{\text {Basis }}=0$ ausgegangen und die Komponenten addiert (GI. (5)).

$$
\begin{aligned}
& \underline{\underline{C}}_{g e s}=\underline{\underline{C}}_{\text {Basis }}+\underline{\underline{C}}_{\underline{c}}+\underline{\underline{C}}_{\text {Gelenk1 }}+\underline{\underline{C}}_{\text {Kopf1 }}+ \\
& \underline{\underline{C}}_{\text {Gelenk2 }}+\underline{\underline{C}}_{K o p f 2}+\underline{\underline{C}}_{s}
\end{aligned}
$$

Somit ergibt sich die Inverse Steifigkeitsmatrix $\underline{\boldsymbol{C}_{g e s}}$ des 3D-Nanotasters mit

$\underline{\boldsymbol{C}}_{\text {ges }}=\left[\begin{array}{lll}c_{x x} & c_{x y} & c_{x z} \\ c_{y x} & c_{y y} & c_{y z} \\ c_{z x} & c_{z y} & c_{z z}\end{array}\right]$

Exemplarisch wird die nominelle Steifigkeit $\mathrm{C}_{\mathrm{zz}}$ des 3D-Nanotasters angegeben, wobei $E$ den E-Modul des Materials beschreibt und $L_{3}=$ $L_{2}+L_{k 2}$ ist.

$$
\begin{aligned}
& C_{z z}=\frac{4 L_{e f f}^{3}}{E w t^{3}}-\frac{4\left(L_{k 1}+L_{1}\right)^{3}}{E w t^{3}}+\frac{4\left(L_{k 1}+L_{1}\right)^{3}}{E w_{1} t_{1}^{3}}-\frac{4 L_{k 1}^{3}}{E w_{1} t_{1}^{3}}+ \\
& \frac{4 L_{k 1}^{3}}{E w t^{3}}-\frac{4\left(L_{k}+L_{2}\right)^{3}}{E w t^{3}}+\frac{4\left(L_{k}+L_{2}\right)^{3}}{E\left(w-w_{k}\right) t^{3}}-\frac{4 L_{e f f}^{3}}{E\left(w-w_{k}\right) t^{3}}+ \\
& \frac{2 L_{2}^{3}}{E w_{3} t^{3}}-\frac{4 L_{e f f}^{2} L_{2}}{E w t^{3}}-\frac{6\left(L_{k 1}+L_{1}\right)^{2} L_{1}}{E w t^{3}}+\frac{6\left(L_{k 1}+L_{1}\right)^{2} L_{1}}{E w_{1} t_{1}^{3}}- \\
& \frac{6 L_{k 1}^{2} L_{3}}{E w_{1} t_{1}^{3}}+\frac{6 L_{k 1}^{2} L_{3}}{E w t^{3}}-\frac{6\left(L_{k}+L_{2}\right)^{2} L_{3}}{E w t^{3}}+\frac{6\left(L_{k}+L_{2}\right)^{2} L_{3}}{E\left(w-w_{k}\right) t^{3}}- \\
& \frac{4 L_{2}^{2} L_{3}}{E\left(w-w_{k}\right) t^{3}}+\frac{4 L_{2}^{2} L_{3}}{E w_{3} t^{3}}+\frac{12 L_{3}^{3}}{E w_{2} t_{2}^{3}}-\frac{12 L_{k 2}^{3}}{E w_{2} t_{2}^{3}}+\frac{4 L_{k 2}^{3}}{E w_{k} t^{3}}- \\
& \frac{6 L_{e f f}^{2} L_{3}}{E w t^{3}}-\frac{6\left(L_{k 1}+L_{1}\right)^{2} L_{3}}{E w t^{3}}+\frac{6\left(L_{k 1}+L_{1}\right)^{2} L_{3}}{E w_{1} t_{1}^{3}}-\frac{6 L_{k 1}^{2} L_{3}}{E w_{1} t_{1}^{3}}+ \\
& \frac{6 L_{k 1}^{2} L_{3}}{E w t^{3}}-\frac{6\left(L_{k}+L_{2}\right)^{2} L_{3}}{E w t^{3}}+\frac{6\left(L_{k}+L_{2}\right)^{2} L_{3}}{E\left(w-w_{k}\right) t^{3}}-\frac{6 L_{2}^{2} L_{3}}{E\left(w-w_{k}\right) t^{3}}+ \\
& \frac{3 L_{2}^{2} L_{3}}{E w_{3} t^{3}}+\frac{12 L_{3}^{2} L_{e f f}}{E w t^{3}}-\frac{12\left(L_{k}+L_{2} L_{3}^{2}\right.}{E w t^{3}}+\frac{12\left(L_{k}+L_{2}\right)_{3}^{2}}{E w_{1} t_{1}^{3}}- \\
& \frac{12 L_{3}^{2} L_{k 1}}{E w_{1} t_{1}^{3}}+\frac{12 L_{3}^{2} L_{k 1}}{E w t^{3}}-\frac{12\left(L_{k}+L_{2}\right) L_{3}^{2}}{E w t^{3}}+\frac{12\left(L_{k}+L_{2}\right) L_{3}^{2}}{E\left(w-w_{k}\right) t^{3}}- \\
& \frac{12 L_{2} L_{3}^{2}}{E\left(w-w_{k}\right) t^{3}}+\frac{6 L_{2} L_{3}^{2}}{E w_{3} t^{3}}+\frac{4 L_{s}}{E d^{2} \pi} \quad(7) \\
& \frac{D}{\mathrm{Di}} \quad
\end{aligned}
$$

Dieses Modell wird mit einer FEM-Simulation (Ansys 18.0) überprüft.

\section{Optimierung und Vergleich mit Finite Elemente Simulation}

Ziel der Optimierung ist, wie bereits beschrieben, eine isotrope Steifigkeit des 3DNanotasters, bei einer ersten Eigenfrequenz von $>50 \mathrm{kHz}$ (Abb. 4), mit ausgewogener möglichst hoher Sensitivität.

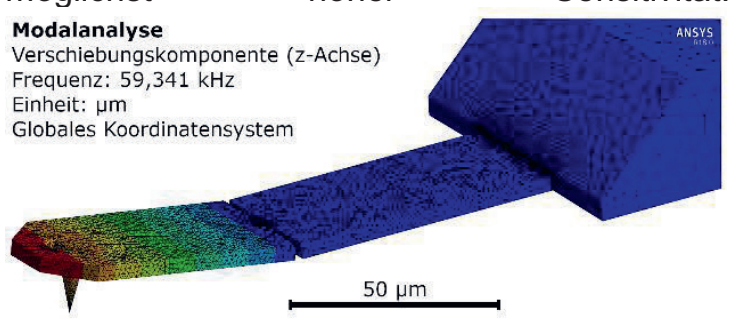

Abb. 4: Grundmode des 3D-Nanotasters

Dementsprechend werden die inversen Steifigkeiten $c_{x x} \approx c_{y y} \approx c_{z z}$ und $c_{y z}=c_{z y}=0$ (Abb. 5), sowie die Auslenkwinkel $\alpha\left(F_{z}\right) \approx 0$ optimiert und $\mathrm{Y}\left(F_{\mathrm{z}}\right)$ minimiert (Abb. 6). 


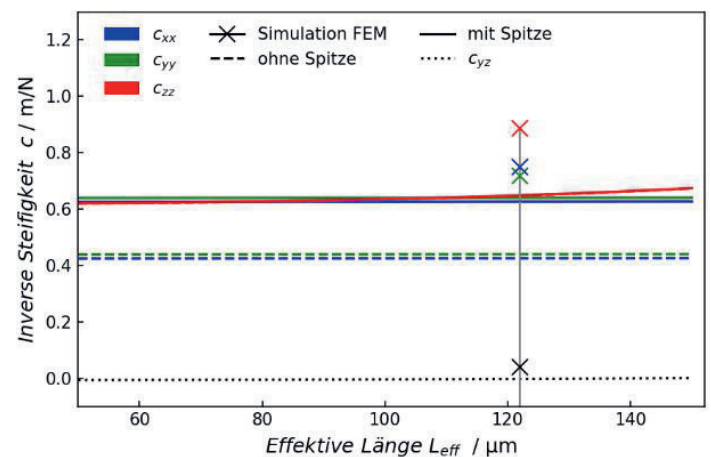

Abb. 5: Inverse Steifigkeiten des Modells und der Simulation über der Effektiven Länge des Cantilevers, mit und ohne der CD-Spitze.

Die Abb. 5 zeigt den Einfluss der sonst die Steifigkeit bestimmenden effektiven Länge des Cantilevers für den 3D-Nanotaster. Der Kurvenverlauf zeigt, dass die mechanischen Eigenschaften der Struktur durch die Festkörpergelenke dominiert wird. Für eine zuvor durch Optimierung festgelegte Struktur, weist die FEM-Simulation eine Abweichung bezüglich der Inversen Steifigkeiten auf. Die inverse Steifigkeit ist insbesondere für die nominelle inverse Steifigkeit erhöht, welches auf die nicht im Modell berücksichtigten elastischen Einspannungen der Festkörpergelenke zurückzuführen ist.

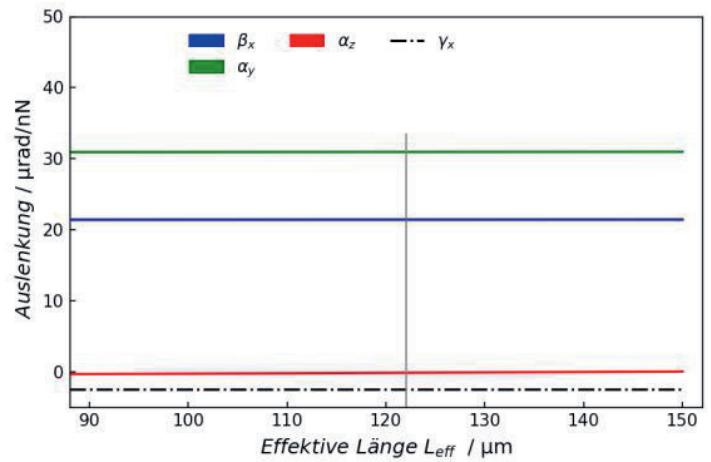

Abb. 6: Winkelauslenkung des Cantilevers für die an der Spitze wirkenden Kräfte bei einem Nanonewton über der effektiven Länge.

Aus Abb. 6 wird ersichtlich das mit dem 3DNanotaster Antastkräfte von weniger als $1 \mathrm{nN}$ detektierbar sein sollten. Die Abbildung zeigt eine ähnliche Auslenkung für an der Spitze wirkende Kräfte, für die $x$ - und $y$-Richtungen, und das zuvor geforderte minimierte Verkippen des Kopfbereiches für eine Kraft in z-Richtung. Die Verbesserung der Eigenschaften ist in Tab. 1 dargestellt.
Tab. 1: FEM-Simulierte* und Modellbasierte Eigenschaften des 3D-Nanotasters im Vergleich zum CD-Cantilever mit $d=64,5 \mathrm{~nm}$

\begin{tabular}{|c|c|c|}
\hline $\begin{array}{c}\text { Eigenschaft } \\
(\mathrm{x} / \mathrm{y} / \mathrm{z})\end{array}$ & CD-Cantilever & 3D-Nanotaster \\
\hline $\begin{array}{c}\text { Sensitivität } \\
\text { in } \mu \mathrm{rad} / \mathrm{nm}\end{array}$ & $(0,28 / 0,50 / 12,0)$ & $(34,2 / 48,4 / 0,21)$ \\
\hline Isotropie & $(7,09 / 7,13 / 1,00)^{*}$ & $(0,84 / 0,81 / 1,00)^{*}$ \\
$\begin{array}{c}\text { Steifigkeits- } \\
\text { verhältnis }\end{array}$ & $(6,68 / 6,71 / 1,00)$ & $(0,97 / 0,99 / 1,00)$ \\
\hline
\end{tabular}

Es wird gezeigt, dass die Isotropie um den Faktor sieben verbessert wurde bei gelichzeitiger 95-fachen Erhöhung der Sensitivität.

Die Grundmode von 59,3 kHz (Material: anisotropes Silizium) wurde mit einer Modalanalyse simuliert.

\section{Diskussion}

Das vorgestellte Design des 3D-Nanotasters zeigt, dass durch modellbasierte Optimierung ein quasi isotroper Taster erzeugbar ist, bei dem die Spitze dezentral angeordnet ist. Jedoch tritt bei dem Vergleich der Simulation zum Modell eine Abweichung auf. Diese Abweichung ist auf den Vergleich eines linearen Modells mit einer nichtlinearen Simulation zurückzuführen, die die Einspannbedingungen der Festkörpergelenke abbildet, wobei der Schubeinfluss vernachlässigbar klein ist. Diese nicht berücksichtigten Einspannbedingungen sind maßgeblich für die Abweichungen in den inversen Steifigkeiten. Es existieren Festkörpergelenke die in ihrer Geometrie einen Übergangsbereich bezüglich des eigentlichen Verformungsbereichs des Gelenkes zeigen und die Relevanz der Einspannung verringern, Fertigungsbedingt wurde jedoch eine rechteckige Form der Festkörpergelenke gewählt. Weiterhin sind aufgrund der Fertigungsabweichung, der einige $100 \mathrm{~nm}$ dicken Strukturen, Abweichungen in dieser Größenordnung von 20\% zu erwarten, so dass dieses Modell für eine Optimierung verwendet werden kann. Die Idee wurde in einem Patent (Nr.: 102018105756 B3) festgehalten.

\section{Zusammenfassung}

Das Modell des isotropen Cantilevers auf Basis eines CD-AFM Cantilevers (CD = Critical Dimension), ist eine Möglichkeit einen 3DNanotaster zu erzeugen. Dabei zeigt der quasi isotrope 3D-Nanotaster eine 95-fach erhöhte Sensitivität im Vergleich zu bisherigen Sonden mit Spitzendurchmessern von nominell $70 \mathrm{~nm}$ (CD-AFM Cantilever). Der 3D-Nanotaster wurde so optimiert, dass sein freies Ende (Kopfbereich) für ein Anzeigesystem benutzt 
werden kann. Eine Kraft senkrecht zu seiner Längsachse und Probenebene in z-Richtung führt zu einer Verschiebung des Kopfbereiches, orthogonal orientierte Kräfte dazu in $x$ - und $y$ Richtung zu einer Verkippung des Kopfbereiches. Somit wäre ein Interferometer mit gekoppelter Quadranten-Photodiode eine Ausleseoption [16]. Die Grundmode des Designs für das Material Silizium liegt bei 59,3 $\mathrm{kHz}$. Das Design findet sich im Patent Nr.: 10 2018105756 B3.

\section{Danksagung}

Spezieller Dank gilt Jannick Langfahl-Klabes für die Aufnahmen im Rasterelektronenmikroskop, sowie der DFG finanzierten Research Training Group 1952 Metrology for Complex Nanosystems, NanoMet und International Graduate School of Metrology B-IGSM.

\section{Literaturnachweis}

[1] G. Binning, C.F. Quate, Ch. Gerber, Physical Review Letters 56, 6 (1986); doi: 10.1103/PhysRevLett.56.930

[2] Y. Matrin, K Wickramasinghe, Appl. Phys. Lett. 64, 2498 (1994); doi: 10.1063/1.111578

[3] G. Dai, M. Heidelmann, C. Kübel, R. Prang, J. Fluegge, H. Bosse, Meas. Sci. Technol., 24, 085001 (2013); doi: 10.1088/09570233/24/8/085001

[4] Team Nanotec GmbH, Wilhelm-Schickardt-Str. 10, 78052 Villingen-Schwenningen, Germany https://www.team-nanotec.de/index.cfm

[5] nanotools $\mathrm{GmbH}$, Reichenbachstraße 33 D - 80469 München https://www.nanotools.com/products/blueline/cdr-ebd/

[6] R. Dixson, N. Orji, I. Misumi, G. Dai, Ultramicroscopy. 194, (2018); doi: 10.1016/j.ultramic.2018.08.011.

[7] V. Nesterov, P. Pornnoppadol, U. Brand, (2003). Metrological characterization of a new three-axis piezoresistive micro probe, Proc. of Euspen 2002 s. 737-740

[8] G. Dai, S. Bütefisch, F. Pohlenz, H.-U. Danzebrink, Measurement Science and Technology, 20. 084001, (2009); doi: 10.1088/0957-0233/20/8/084001

[9] J. Claverley, R. Leach, (2011). Verifying the capability of a three axis vibrating micro-scale CMM probe to operate in a non-contact mode. Proceedings - ASPE 2011 Annual Meeting. 52.

[10]J. Cui, J. Li, K. Feng, J. Tan, Optics Express. 23. 26386-26398. (2015); doi: 10.1364/OE.23.026386.

[11] K. Alblalaihid, et. al., Sensors. 16. 492. (2016); doi: 10.3390/s16040492

[12]L. Zou, H. Ni, P. Zhang, X. Ding, Sensors (Basel, Switzerland), 17(11), 2652 (2017); doi:10.3390/s17112652
[13] R.-L. Li, P. Xu, P.-Y. Wang, K.-C. Fan, R.-J. Cheng, (2018). Sensors. 18. 4229. (2018); doi: 10.3390/s18124229

[14] BS Precision Engineering Deutschland $\mathrm{GmbH}$, Leitzstraße 45, 70469 Stuttgart, Germany http://www.ibspe.com/category/triskelion-touchprobe.htm

[15] Werth Messtechnik GmbH, D-35394 Gießen https://www.werth.de/fileadmin/media/pdf/Facha rtikel/FA-

Messen_von_Mikrogeometrien_mit_dem_Faser taster-Mikroproduktion_02.2016.pdf

[16] Felix G Balzer et al, Meas. Sci. Technol. 22 094018, (2011); doi:10.1088/09570233/22/9/094018

[17] G. Jäger, E. Manske, T. Hausotte, A.Müller, F. Balzer, Surf. Topogr.: Metrol. Prop.4 034004 (2016); doi: 10.1088/2051-672X/4/3/034004 